\title{
MANEJO CIRÚRGICO DA SÍNDROME DE MIRIZZI
}

\author{
Surgical management of Mirizzi syndrome
}

Olival Cirilo Lucena da FONSECA-NETO, Maria das Graças Lapenda PEDROSA, Antonio Lopes MIRANDA

ABCDDV/591

Fonseca-Neto OCL, Pedrosa MGL, Miranda AL. Manejo cirúrgico da síndrome de Mirizzi. ABCD Arq Bras Cir Dig. 2008;21(2):51-4

RESUMO - Racional - Síndrome de Mirizzi é rara condição encontrada em pacientes com colelitíase de longa data, variando de 0,3 - 3\% nos pacientes submetidos à colecistectomia. Se não reconhecida no pré-operatório pode implicar em significativa morbimortalidade. Objetivo - Descrever série de cinco pacientes consecutivos com síndrome de Mirizzi submetidos à cirurgia e comentar as suas características clínicas. Método - Revisão retrospectiva de cinco pacientes com síndrome de Mirizzi, entre janeiro de 2002 e junho de 2008. Foram avaliados: a apresentação clínica, resultados laboratoriais, avaliação pré-operatória, achados cirúrgicos, presença de coledocolitíase, classificação da síndrome de Mirizzi, escolha do procedimento operatório e suas complicações. Resultados - Quatro pacientes eram mulheres (80\%) e a média de idade foi 53,4 anos (38 a 62 anos). Os sintomas mais freqüentes foram dor abdominal (100\%) e náuseas / vômitos (100\%). Todos os pacientes com icterícia apresentaram alterações da função hepática $(40 \%)$ e apenas um, sem icterícia, tinha bioquímica hepática alterada. O diagnóstico de síndrome de Mirizzi foi intra-operatório em todos $(100 \%)$ casos. A associação entre fístula coledocociana e coledocolitíase foi observada em três pacientes (60\%). Quanto à classificação, encontrou-se dois pacientes com tipo I e um paciente em cada um dos tipos II, III, IV. A colecistectomia foi realizada em todos os pacientes, sendo parcial em três $(60 \%)$. A anastomose coledocoduodenal foi realizada em dois pacientes, sendo do tipo látero-lateral. A coledojejunoanastomose ocorreu em um único caso (tipo IV). Evolução pós-operatória sem alterações ocorreu em dois casos (40\%) recebendo alta em boas condições. Um paciente apresentou sepse no pós-operatório secundário a abscesso subhepático evoluindo ao óbito no $2^{\circ}$ dia de pós-relaparotomia. Conclusão Apesar do diagnóstico pré-operatório ser raro nos pacientes com síndrome de Mirizzi, ela deve ser suspeitada na colelitíase crônica e prontamente identificada no intra-operatório para evitar lesões biliares inadvertidas. Apesar da era da colecistectomia laparoscópica, o método aberto deve ser o de escolha.

DESCRITORES - Injúria do ducto biliar. Bypass biliar. Mirizzi. Colecistectomia. Cirurgia biliar.

\section{INTRODUÇÃO}

A síndrome de Mirizzi é a obstrução do ducto hepático comum secundária a compressão extrínseca devido a impactação de cálculos no ducto cístico ou no infundíbulo da vesícula1. É complicação não-usual da doença litíasica da vesícula biliar e ocorre em aproximadamente $1 \%$ de todos os pacientes com colelitíase ${ }^{20}$.

A importância dela deriva do alto risco de lesões do ducto biliar durante os procedimentos cirúrgicos. Soma-se a esse fato a dificuldade do diagnóstico antes da operação. Em pacientes ictéricos ou não, operações de emergência e escassos recursos radiológicos proporcionam condição propícia para lesões iatrogênicas da via biliar.

O objetivo deste estudo é descrever uma série de cinco pacientes com síndrome de Mirizzi, submetidos a tratamento cirúrgico, e comentar os aspectos etiopatogênicos e apresentação clínica, enfatizando o diagnóstico e o tratamento dessa complicação da litíase biliar.

Trabalho realizado no Serviço de Cirurgia Geral e do Trauma do Hospital da Restauração - SUS, Recife, PE, Brasil

Endereço para correspondência: Olival Cirilo Lucena Fonseca Neto. E-mail: olivalneto@globo.com

\section{MÉTODO}

Cinco pacientes consecutivos foram operados e diagnosticados com síndrome de Mirizzi entre janeiro de 2002 e junho de 2008, pelos autores em três hospitais públicos do Recife, PE (Hospital Geral Otávio de Freitas, Hospital Universitário Oswaldo Cruz e Hospital da Restauração).

As informações clínicas e morfológicas foram obtidas através de consulta aos prontuários médicos dos pacientes incluídos no estudo e acompanhados até esta data no ambulatório do Hospital Universitário Oswaldo Cruz (HUOC).

O critério de inclusão foi a caracterização da síndrome de Mirizzi pela presença de colecistolitíase e dilatação do ducto hepático comum acima do nível da obstrução causada por cálculo impactado no ducto cístico ou no infundíbulo da vesícula biliar.

Foram avaliados a apresentação clínica; os resultados de laboratório e exames de imagem; os achados operatórios; a presença de coledocolitíase; a classificação de acordo com Csendes et al. ${ }^{5}$ (Tabela 1, Figura 1); a escolha do procedimento operatório; e as complicações pré-operatórias. 
TABELA 1 - Classificação da síndrome de Mirizzi de acordo com Csendes et al. ${ }^{5}$

\begin{tabular}{ll}
\hline Tipo & Características \\
\hline Tipo I & $\begin{array}{l}\text { Obstrução extrínseca do ducto hepático } \\
\text { comum, cálculos geralmente impactados } \\
\text { no ducto cístico ou no infundíbulo da } \\
\text { vesícula } \\
\text { Presença de fístula colecistobiliar com } 1 / 3 \\
\text { do diâmetro da circunferência do ducto } \\
\text { hepático comum } \\
\text { Tipo II } \\
\text { Tipo III } \\
\text { mais de } 2 / 3 \text { do diâmetro da circunferência } \\
\text { do ducto hepático comum } \\
\text { Presença de fístula colecistobiliar que } \\
\text { envolve toda a circunferência do ducto } \\
\text { hepático comum }\end{array}$ \\
\end{tabular}

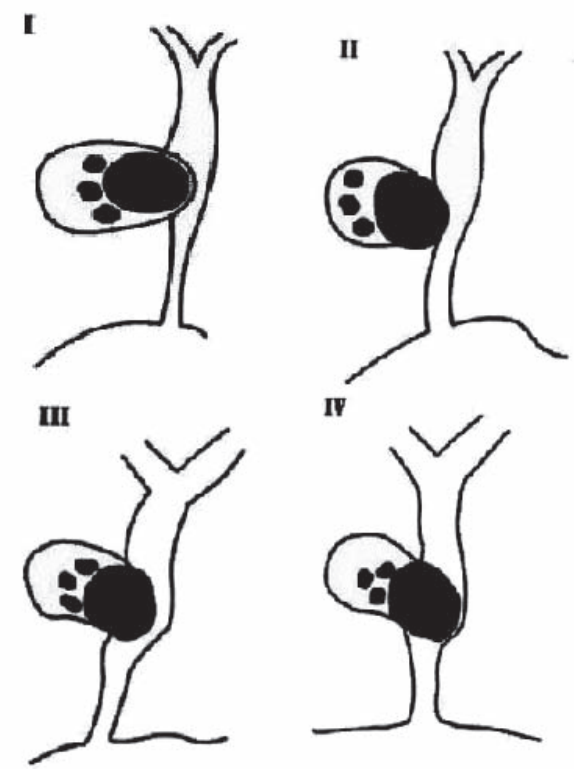

FIGURA 1 - Representação esquemática da classificação de Csendes $^{5}$ para a síndrome de Mirizzi

\section{RESULTADOS}

Quatro pacientes eram do sexo feminino (80\%). A idade variou de 38 a 62 anos. Três eram da raça negra, um da raça branca e um, amarela (Tabela 2). Os sinais e sintomas mais freqüentes estão mostrados na Tabela 3.

Os exames da função hepática estavam alterados em $50 \%$ dos pacientes. As enzimas canaliculares (fosfatase alcalina e gamaglutamiltranspeptidade) foram as que mais chamaram a atenção. Os seus valores flutuaram 3 e 5 vezes os valores normais (220 - $720 \mathrm{UI} / \mathrm{L})$. A hiperbilirrubinemia foi flagrada em $40 \%$ dos casos e os seus valores variaram de 2 a $7,5(\mathrm{mg} / \mathrm{dL})$.

O diagnóstico foi intra-operatório em todos os casos. Em três pacientes, a ultrassonografia foi realizada e revelou colelitíase e dilatação da via biliar intra-hepática. Em um caso, a ultrassonografia sugeriu cisto de colédoco. Neste paciente foi realizada tomografia que mostrou colelitíase e coledocolitíase com dilatação da via biliar principal. A colangiografia através da endoscopia retrógrada não foi utilizada em nenhum caso.
TABELA 2 - Características clínicas, diagnósticas e operatórias dos pacientes com síndrome de Mirizzi.

\begin{tabular}{|c|c|c|c|c|c|c|c|}
\hline Caso & Sex & Idade & e Raça & Icterícia & Classificação & Coledocolitiase & Tipo de operação \\
\hline 1 & $\mathrm{~F}$ & 38 & Amarela & Não & II & Sim & $\begin{array}{l}\text { Colecistectomia }+ \text { co- } \\
\text { ledocoduodenoanasto- } \\
\text { mose }\end{array}$ \\
\hline 2 & M & 62 & Branca & Sim & III & Sim & $\begin{array}{c}\text { Colecistectomia parcial } \\
+ \text { coledocoduodeno- } \\
\text { nastome }\end{array}$ \\
\hline 3 & $\mathrm{~F}$ & 57 & Negra & Não & I & Não & $\begin{array}{l}\text { Colecistectomia + colo- } \\
\text { cação de dreno de Kehr }\end{array}$ \\
\hline 4 & $\mathrm{~F}$ & 51 & Negra & Sim & IV & Sim & $\begin{array}{c}\text { Colecistectomia par- } \\
\text { cial + coledocoplastia }+ \\
\text { coledocojejunoanasto- } \\
\text { mose em Y de Roux }\end{array}$ \\
\hline 5 & $\mathrm{~F}$ & 59 & Negra & Não & I & Não & colecistectomia parcial \\
\hline
\end{tabular}

TABELA 3 - Sinais e sintomas mais freqüentes presentes nos pacientes com síndrome de Mirizzi.

\begin{tabular}{lcc}
\hline Sinais ou sintomas & N & \% \\
\hline Dor abdominal & 5 & $100 \%$ \\
Icterícia & 2 & $40 \%$ \\
Náuseas e vômitos & 5 & $100 \%$ \\
Colúria & 2 & $40 \%$ \\
Febre & 4 & $80 \%$ \\
\hline
\end{tabular}

A incisão cirúrgica utilizada foi a subcostal direita e a colecistectomia pelo método aberto foi a escolhida em todos os casos.

Os achados intra-operatórios mostraram cálculo na vesícula biliar (no infundíbulo ou no cístico). Fístula colecistobiliar associada com colelitíase foi observada em três situações (60\%). A colangiografia intra-operatória foi realizada em um caso $(20 \%)$.

Quanto à classificação, foram encontrados: tipo I em $40 \%$, tipo II $20 \%$, tipo III $20 \%$ e tipo IV em $20 \%$.

Dois pacientes apresentaram infecções do sítio cirúrgico superficial que foram tratados com abertura da ferida operatória e limpeza manual. Evoluíram com melhora após o $15^{\circ}$. dia do pós-operatório. Um paciente (n 2), apresentou sepse no $8^{\circ}$. dia de pós-operatório devido a abscesso subhepático. Foi operado e drenado. A disfunção de múltiplos órgãos ocorreu no $2^{\circ}$. dia de pós-operatório da relaparotomia e evoluiu para o óbito.

\section{DISCUSSÃO}

Em 1948, Mirizzi descreveu uma síndrome hepática funcional no qual consistia na obstrução do ducto hepático comum secundária a compressão de cálculos biliares impactados no ducto cístico ou no infundíbulo da vesícula biliar. Inflamação, colangite de repetição e espasmo do esfíncter circular do ducto hepático também faziam parte dos achados desta síndrome $\mathrm{e}^{6,7,12,16}$.

Em 1982, McSherry ${ }^{13}$ sugere subclassificar a síndrome de Mirizzi em dois tipos: I, onde existe compressão externa do ducto hepático comum por cálculo no cístico ou na bolsa de Hartman, da vesícula biliar; II, onde o cálculo erodiu parcialmente ou completamente a parede do ducto biliar 
comum resultando em fístula colecistocoledociana ${ }^{13}$.

Csendes et al. ${ }^{5}$ sugeriram nova classificação baseada no tamanho da fístula biliar. As lesões do tipo I seriam aquelas devido à compressão externa da via biliar principal; as do tipo II, onde a fístula colecistobiliar erodiu menos do que $1 / 3$ da circunferência do ducto biliar; as do tipo III, onde a fístula envolveu mais do que $2 / 3$ da circunferência do ducto biliar e as do tipo IV quando existisse destruição completa do ducto biliar.

Nos últimos 20 anos vários autores descreveram diferentes sistemas de classificação para os pacientes com síndrome de Mirizzi, como: aguda vs crônica; variação anatômica do ducto cístico vs sem variação do ducto cístico; obstrução devido a cálculo vs obstrução devido à inflamação ${ }^{14,15,21}$. As classificações de McSherry ${ }^{13}$ e Csen$\operatorname{des}^{5}$ são as mais utilizadas, por melhor guiar o manejo cirúrgico. A incidência reportada do tipo I de McSherry varia de 11 a $45 \%$ e a tipo II McSherry (Csendes II, III e IV) varia de 55 a $89 \%$. Apenas $6 \%$ dos pacientes com síndrome de Mirizzi têm Csendes tipo IV. Neste estudo, os pacientes obedeceram a freqüência de distribuição da classificação como sugere: tipo I $20 \%$, tipo II $80 \%$ (McSherry) ${ }^{13}$.

O diagnóstico pré-operatório da síndrome de Mirizzi é muito importante devido a associação com lesões de via biliar iatrogênicas ${ }^{9}$. Nos últimos cinco anos mais de 76 artigos foram publicados com referência a síndrome de Mirizzi (Medline Consultonat). Esse número de artigos é bem maior do que os publicados em 50 anos da descrição original dessa síndrome.

Em menos de $50 \%$ dos casos o diagnóstico é feito previamente à operação (geralmente ocorrendo em unidades especializadas). A icterícia e a dor abdominal podem ocorrer em 60 a $100 \%$ dos pacientes ${ }^{10,11}$. A ultrassonografia do abdome deve ser usada na triagem inicial e algumas vezes pode mostrar achados típicos de síndrome de Mirizzi, como cálculo grande imóvel no infundíbulo da vesícula biliar e dilatação do ducto biliar acima da obstrução mas normal abaixo dela ${ }^{3,4}$. Tomografia computadorizada (TC) pode ser utilizada, entretanto, os sinais radiológicos não são específicos ${ }^{17}$. A colangioressonância e a TC podem ajudar a diferenciar a síndrome de Mirizzi de malignidades do sistema biliar extrahepático ${ }^{19}$. A colangiografia endoscópica retrógrada mostrará as anormalidades dos ductos biliares e a fístula, entretanto, pode apresentar algumas complicações ${ }^{22}$. Nestes pacientes, o diagnóstico foi intra-operatório em $100 \%$ dos casos. A ultrassonografia abdominal foi realizada em três pacientes que não sugeriu síndrome de Mirizzi. A TC foi realizada em um que apenas afastou a presença de malignidade na árvore biliar extra-hepática.
A colecistectomia anterógrada convencional é perigosa nesses pacientes. A do tipo retrógrada (iniciada pelo fundo vesicular) deve ser a escolhida, porém, não deve resolver totalmente o problema. A colecistectomia parcial foi descrita em 1985, para "as vesículas biliares difíceis" (mais na presença da hipertensão portal) e pode ser utilizada com segurança nesses pacientes ${ }^{4}$. A investigação da causa da icterícia através de colangiografia intra-operatória é aconselhada. A drenagem da via biliar principal com tubo- $T$ pode ser necessária e segura em casos específicos. É importante reconhecer a presença da fistula colecistobiliar para não permanecer dissecando entre o espaço inexistente entre vesícula e ducto hepático comum. Csendes et $\mathrm{al}^{5}$, estudando série de 219 pacientes recomenda como manejo cirúrgico colecistectomia parcial e retirada dos cálculos para ver o ducto biliar comum e definir o tipo e a localização da fístula com colangiografia intra-operatória. Esse autor aconselha colocar tubo-T se o processo inflamatório/fibrótico for intenso e a colangiografia não for realizada. Coledocoplastia ou sutura da fístula com material absorvível também foi recomendada (tipo II e III). No tipo IV a anastomose bilioentérica é preferida. Nesta série, o tratamento cirúrgico foi baseado no achado cirúrgico sem colangiografia. A colecistectomia foi realizada no primeiro tempo, mas nem sempre foi conseguida (60\% - colecistectomia parcial). A drenagem a Kehr (n 3) foi optada pelo quadro de inflamação intensa (operada de emergência - colecistite aguda). A anastomose biliodigestiva ocorreu em $60 \%$ dos casos, sendo a coledocojejunoanastomose em um único caso do tipo IV (n 4). Apesar do diagnóstico intra-operatório, não foi observada lesão inadvertida da via biliar nessa série de casos.

Complicações são encontradas em $0-60 \%$ variando desde infecção do sítio cirúrgico a fístulas e estenoses biliares $^{18}$. A mortalidades hospitalar é reportada entre $0-25 \% 8$. Este estudo mostrou dois casos de infecção de sítio cirúrgico superficial que melhoraram com abertura ampla e limpeza manual. Óbito ocorreu em um paciente devido à sepse de origem abdominal (abscesso subhepático).

\section{CONCLUSÃO}

Apesar do diagnóstico pré-operatório ser raro nos pacientes com síndrome de Mirizzi, ela deve ser suspeitada na colelitíase crônica e prontamente identificada no intraoperatório para evitar lesões biliares inadvertidas. Apesar da era da colecistectomia laparoscópica, o método aberto deve ser o de escolha. 
Fonseca-Neto OCL, Pedrosa MGL, Miranda AL. Surgical management of Mirizzi syndrome.ABCD Arq Bras Cir Dig. 2008;21(2):51-4

ABSTRACT - Background - Mirizzi syndrome is a rare complication of long standing cholelithiasis and was reported in 0,3 - 3\% of patients undergoing cholecystectomy. If not recognized preoperatively, it can result in significant morbidity and mortality. Aim - To describe a series of five consecutive patients with Mirizzi syndrome submitted to surgical treatment and to comment on then aspects clinics. Methods - A retrospective review of five consecutives cases of Mirizzi syndrome that arose between January 2002 and June 2008 was performed. The following items were evaluated: clinical presentation, laboratory results, preoperative evaluation, operative findings, presence of choledocholithiasis, type of Mirizzi syndrome according to the classification by Csendes, choice of operative procedures, and complications. Results - Four patients were women $(80 \%)$ and a mean age was 53,4 years (38 to 62 years. The most frequent symptoms were abdominal pain (100\%) and nausea and vomiting (100\%) The patients with jaundice presented altered hepatic function tests $(40 \%)$ and only one without jaundice presented altered hepatic function. The diagnosis of Mirizzi syndrome was intra-operative in all patients (100\%). Cholecystecholedochal fistula associated with choledocholithiasis was observed in three $(60 \%)$ cases. Mirizzi syndrome was classified as Csendes type I in two (40\%) patients, type II in one (20\%), type III in one $(20 \%)$ and type IV in another $(20 \%)$. Cholecystectomy was performed in all patients $(100 \%)$, however, the partial cholecystectomy was observed in three $(60 \%)$. Two $(40 \%)$ patients were submitted to side-to-side choledochoduodenostomy and another $(20 \%)$ to choledochojejunoanastomosis. Two (40\%) patients had an uneventful recovery and were discharged in good conditions. One (20\%) patient presented a postoperative sepsis due to a sub-hepatic abscess and was reoperated. This patient to die. Conclusions - The preoperative diagnosis of Mirizzi syndrome is a challenge. A high index of clinical suspicion is required to make an intra-operative diagnosis, which leads to good surgical planning to treat the condition. Open surgery is the gold standard.

HEADINGS - Bile duct injury. Biliary bypass. Mirizzi. Cholecystectomy. Biliary surgery.

\section{REFERÊNCIAS}

1. Artifon ELA, Sakai P, Hondo FY, Noda RW, Ishioka S. Mirizzi syndrome type IV: a rare entity. Digestive Endoscopy 2003; 15:344-347.

2. Becker CD, Grossholz M, Becker M, Mentha G, de Peyer R, Terrier F. Choledocholithiasis and bile duct stenosis: diagnostic accuracy of MR cholangiopancreatography. Radiology. 1997 Nov;205(2):523-30.

3. Becker CD, Hassler H, Terrier F. Preoperative diagnosis of the Mirizzi syndrome: limitations of sonography and computed tomography. AJR Am J Roentgenol 1984; 143:591-6.

4. Bornman PC, Terblanche J. Subtotal cholecystectomy: for the difficult gallbladder in portal hypertension and cholecystitis. Surgery 1985; 98:1-6.

5. Csendes A, Díaz JC, Burdiles P, Maluenda F, Nava O. Mirizzi syndrome and cholecystobiliary fistula: a unifying classification. Br J Surg. 1989 Nov; 76(11):1139-43

6. Estale E. [Pablo L. Mirizzi]. Prensa Med Argent 1964; 51:845-8.

7. Estale E. [Pablo L. Mirizzi]. Prensa Med Argent 1967; 54:939-940.

8. Fletcher DR, Hobbs MS, Tan P et al. Complications of cholecystectomy: risks of the laparoscopic approach and protective effects of operative cholangiography. Ann Surg 1999; 229:449-57.

9. Gomez G. Mirizzi syndrome. Curr Treat Options Gastroenterol 2002; $5: 95-9$.

10. Ibrarullah MD, Saxena R, Sikora SS, Kapoor VK, Saraswat VA, Kaushik SP. Mirizzi's syndrome: identification and management strategy. Aust N Z J Surg 1993; 63:802-6.

11. Johnson LW, Sehon JK, Lee WC, Zibari GB, McDonald JC. Mirizzi's syndrome: experience from a multi-institutional review. Am Surg 2001; 67(1):11-14.

12. Leopardi LN, Maddern GJ. Pablo Luis Mirizzi: the man behind the syndrome. ANZ J Surg 2007; 77:1062-1064.
13. McSherry CK, Fertenberg H, Virshup M. The Mirizzi syndrome: suggested classification and surgical therapy. Surg Gastroenterol 1982; 1:219-25.

14. Morelli A, Narducci F, Ciccone R. Can Mirizzi syndrome be classified into acute and chronic form? An endoscopic retrograde cholangiography (ERC) study. Endoscopy 1978; 10:109-12.

15. Nagakawa T, Ohta T, Kayahara M, Ueno K, Konishi I, Sanada H. A new classification of Mirizzi syndrome from diagnostic and therapeutic viewpoints. Hepatogastroenterology 1997; 44:63-7.

16. No authors listed. Pablo Luis Mirizzi (1893-1964)]. Bull Soc Int Chir 1964; 23:479-81.

17. Pedrosa CS, Casanova R, de la Torre S, Villacorta J. CT findings in Mirizzi syndrome. J Comput Assist Tomogr 1983; 7:419-25.

18. Posta CG. Unexpected Mirizzi anatomy: a major hazard to the common bile duct during laparoscopic cholecystectomy. Surg Laparosc Endosc 1995; 5:41214.

19. Ravo B, Epstein H, La Mendola S, Ger R. The Mirizzi syndrome: preoperative diagnosis by sonography and transhepatic cholangiography. Am J Gastroenterol 1986; 81:688-90.

20. Safioleas M, Stamatakos M, Safioleas P, Smyrnis A, Revenas C, Safioleas C. Mirizzi syndrome: na unexpected problem of cholelithiasis. Our experience with 27 cases. Int Semin Surg Oncol 2008; 5:12.

21. Starling JR, Matallana RH. Benign mechanical obstruction of the common hepatic duct (Mirizzi syndrome). Surgery 1980; 88:737-40.

22. Tan KY, Ching HC, Chen CYY, Tan SM, Poh BK, Hoe MNY. Mirizzi syndrome: noteworthy aspects of a retrospective study in one centre. ANZ J Surg 2004; 74:833-7. 